\title{
Regulação do trabalho médico no Brasil: impactos na Estratégia Saúde da Família
}

\author{
I ${ }^{1}$ Paulo Henrique de Almeida Rodrigues, ${ }^{2}$ Márcia Silveira Ney, \\ ${ }^{3}$ Carlos Henrique Assunção Paiva, ${ }^{4}$ Luciana Maria Borges da Matta Souza I
}

Resumo: $\mathrm{O}$ artigo apresenta uma revisão da regulação do trabalho dos médicos de família no Brasil e em outros países, com o objetivo de discutir medidas recentes do Ministério da Saúde que flexibilizaram a carga horária de trabalho dos médicos na Estratégia Saúde da Família. A abordagem é feita a partir de uma revisão bibliográfica e da legislação brasileira sobre o tema, numa perspectiva comparada com experiências de outros países. A pesquisa revelou a existência de um padrão de baixa regulação estatal tanto do trabalho médico, quanto de sua formação no Brasil, especialmente no que diz respeito à medicina da família, quando comparada com experiências internacionais. Esta situação resulta numa baixa oferta de profissionais para a Estratégia Saúde da Família e contribuiu para a recente flexibilização da carga horária dos médicos e para a criação do Programa Mais Médicos pelo Ministério da Saúde. A opção pela flexibilização no lugar de maior regulação sobre a profissão pode afetar a integração das equipes de saúde da família, que constitui elemento central da estratégia e aparentemente contradiz a ênfase no papel prioritário da mesma reiteradamente declarada pelo ministério.

> Palavras-chave: Saúde da Família; Medicina de Família e Comunidade; recursos humanos em saúde; políticas públicas de saúde.

\author{
1 Sociólogo; professor adjunto \\ do Instituto de Medicina Social \\ da Universidade do Estado do \\ Rio de Janeiro e do Mestrado \\ em Saúde da Família da \\ Universidade Estácio de Sá. \\ Rio de Janeiro, Brasil. Endereço \\ eletrônico: phphysis@gmail.com

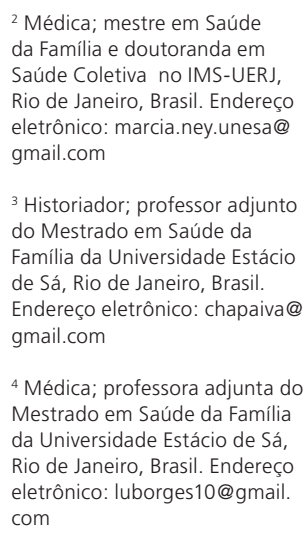

${ }^{4}$ Médica; professora adjunta do Mestrado em Saúde da Família da Universidade Estácio de Sá, Rio de Janeiro, Brasil. Endereço eletrônico: luborges10@gmail. com

Recebido em: 22/05/2013 Aprovado em: 28/11/2013 
Diversos estudiosos apontam desafios e expectativas em torno da implantação da Estratégia Saúde da Família (ESF), vista como palco privilegiado para experimentação e desenvolvimento de práticas preventivas e assistenciais afinadas ao princípio da integralidade da atenção, tal como formulado pelo inciso II, do parágrafo $7^{\circ}$ da Lei no 8.080 (VIANA; DAL POZ, 2005; GIL, 2006; FEUERWERKER, 2005; ESCOREL et al., 2007). Iniciada em 1994, a ESF pode ser considerada uma das mais importantes políticas públicas de atenção à saúde, que atingiu um total de 33.533 equipes em junho de 2012, segundo dados do Departamento de Atenção Básica (DAB) do Ministério da Saúde, o que equivaleria a uma cobertura de $53,91 \%$ da população brasileira. ${ }^{1}$

Desde 1997, o Ministério da Saúde considera a Saúde da Família uma estratégia voltada para a reorientação do modelo assistencial do Sistema Único de Saúde (SUS) como um todo (BRASIL, 1997). A ESF pretende realizar uma ruptura com as práticas tradicionais desenvolvidas por diferentes trabalhadores do SUS e para a mudança dos processos de trabalho das Equipes de Saúde da Família (ESFs), de forma afinada às necessidades das populaçôes por elas atendidas.

O sucesso da ESF depende de sua capacidade em atrair e manter profissionais de saúde nas suas equipes. Isto tem sido um grande desafio particularmente em relação aos médicos (NEY, 2009; GIRARDI; CARVALHO, 2002; BRASIL, 2002). Uma das razões dessa dificuldade decorre da insuficiente regulação estatal sobre a formação profissional e o exercício da profissão médica no país, inclusive sobre a formação para as especialidades. Não existe em nosso país controle público sobre o "estoque"2 e a formação das diferentes especialidades médicas, tal como ocorre na Austrália, França, Reino Unido e Suécia, com base no pressuposto de que o mercado de saúde não é livre (BLOOR; MAYNARD, 2003, p. 8 e 14). Espanha, Portugal e Canadá e outros que investem nesse tipo de política procuram investir na formação de uma proporção adequada de médicos de família. Segundo a Organização para a Cooperação e o Desenvolvimento Econômico (OCDE), em 2005, tal proporção chegava a 52,1\% na Austrália; na França, 49,3\%; em Portugal, 49\% e no Canadá, 48\% (OECD, 2011).

A dificuldade para atrair e fixar médicos na Saúde da Família decorre, em parte, dessa lacuna da regulação sobre a profissão, apesar da retórica ministerial a respeito da importância da estratégia. Dentre os pontos críticos relativos à fixação 
desse profissional estão: a dedicação de 40 horas semanais; a formação para a especialidade de Medicina de Família e Comunidade e a regulação do mercado de trabalho, temas desenvolvidos neste artigo.

Em outubro de 2011, a Portaria GM/MS no 2.488/2011 - Política Nacional de Atenção Básica (BRASIL, 2011) - alterou normas anteriores quanto à carga de trabalho dos profissionais médicos que compõem as ESFs e ao repasse dos incentivos financeiros relativos a uma equipe, que integra o Piso de Atenção Básica Variável, de forma a flexibilizar a carga de trabalho, visando facilitar a incorporação de profissionais médicos às equipes. Este artigo questiona a eficácia da medida em função: da natureza do mercado de trabalho capitalista; das características sociais da profissão médica; da frágil regulação do trabalho médico no Brasil; dos problemas relacionados às dificuldades para a incorporação e manutenção dos profissionais médicos na ESF; e dos possíveis efeitos da medida sobre o trabalho de equipe e as reações das outras profissões de saúde nela envolvidas.

A partir de julho de 2013, ${ }^{3}$ o Governo Federal criou o Programa Mais Médicos, inicialmente através de uma Medida Provisória (MP no 621, de 8 de julho de 2013) e de uma Portaria Interministerial (Saúde e Educação), publicada no mesmo dia (Portaria Interministerial no 1.369). A Medida Provisória foi depois convertida em Lei (Lei no 12.871, de 22 de outubro de 2013). O programa tem entre os objetivos declarados a diminuição da carência de médicos em regiôes prioritárias para o SUS; o fortalecimento da prestação de serviços de atenção básica e o aprimoramento da formação médica no país. Trata-se de uma iniciativa que visa claramente a maior regulação do Estado sobre a profissão médica, tendo sido recebido de forma bastante crítica por entidades representativas dessa categoria profissional. Seus resultados só poderão ser bem avaliados pelos analistas no futuro.

\section{A profissão médica e a regulação do mercado de trabalho}

Não cabe no escopo deste artigo fazer uma revisão ampla do tema da regulação do trabalho médico. Desta forma, apresentamos apenas alguns pontos considerados relevantes para a abordagem do tema.

Desde o início do capitalismo, o Estado estabeleceu crescentes mecanismos de regulação do trabalho, restringindo, em consequência, os mecanismos de autorregulação até então existentes, como as guildas medievais, através da legislação estatal sobre o trabalho (POLANYI, 1998). Ainda segundo o mesmo 
autor, a ideia de "trabalho livre" só surgiu na Inglaterra a partir do fortalecimento do liberalismo econômico, que se seguiu à Revolução Industrial. A superação do liberalismo econômico que se iniciou a partir da Grande Depressão de 1929 constituiu o pano de fundo para a reintrodução de regras estatais sobre o trabalho no mundo capitalista (BERMAN, 2006; LIMONCIC, 2009; ELEY, 2005; JUDT, 2008). O auge da regulação do trabalho pelo Estado coincidiu com o período denominado por Hobsbawn (1995) como "a era de ouro" do capitalismo -1945 a 1975. A partir dos anos 1980, entretanto, com a ascensão do neoliberalismo, entramos numa fase clara de desregulamentação do mundo do trabalho. Ao contrário do que ocorreu para a maioria das profissōes, a categoria médica conseguiu assegurar maior dose de autonomia do que outras profissões, além de baixo padrão de intervenção do Estado em suas atividades, desde o início da institucionalização de sua profissão (FREIDSON, 2009; MENELEU NETO, 2006; BLOOR; MAYNARD, 2003; RIBEIRO; SCHRAIBER, 1994).

Segundo diversos autores clássicos da sociologia da profissão médica (FREIDSON, 2009; WILLIS, 1983; STARR, 1982), um dos fatores que contribuíram para a autonomia dessa profissão foi a valorização do conhecimento técnico e a grande diversidade de especializaçóes, características que conferem a esses profissionais maior autonomia sobre seu próprio trabalho. A profissão se caracteriza, ainda, pelo fato de os padrões seguidos pelos profissionais no exercício de suas funções - diretrizes clínicas e protocolos - serem oriundos das sociedades médicas especializadas, ou seja, de fora das organizações onde eles trabalham, o que não ocorre em praticamente nenhum outro setor de atividade (MINTZBERG, 1995; FREIDSON, 2009). A valorização do conhecimento técnico, a elevada e diversificada especialização do mesmo, a autonomia no controle do processo de trabalho e os padróes de atividade originados das sociedades profissionais autorreguladas explicam uma singularidade das organizaçōes de saúde, que é a grande influência que os médicos detêm no processo de tomada das decisões e na hierarquia organizacional das organizações de prestação de serviços de saúde. Isto acarreta uma relativa descentralização e democratização das estruturas administrativas, em benefício do médico, ao contrário do que ocorre na maior parte das outras organizações humanas (MINTZBERG, 1995).

Outro aspecto que deriva do controle sobre o conhecimento pelos médicos é a grande influência que eles têm sobre a formação da demanda por bens e serviços 
no setor de saúde. $\mathrm{O}$ aval que as sociedades médicas conseguiram do Estado para o exercício da profissão na maior parte dos países lhes conferiu a prerrogativa da prescrição dos medicamentos, exames diagnósticos e procedimentos terapêuticos para os pacientes, induzindo a maior parte da demanda de serviços (SCHWEITZER, 2007; FELDSTEIN, 1998; STARR, 1982).

Tal prerrogativa também tem sido conseguida através das sociedades médicas especializadas, que influenciam a entrada de novos profissionais no mercado por meio do estabelecimento de exigências para a formação e de restrições ao exercício da prática profissional (IUNES, 1995). Tais associações desempenham papel-chave em todo o mundo no funcionamento dos sistemas e serviços de saúde, assim como no mercado de trabalho (BOERMA; RICO, 2006). Pode-se depreender que situações inerentes às condições do exercício do trabalho no setor não se alterarão de forma "natural", apenas se houver a introdução de mecanismos de regulação, que só o Estado pode fazer.

Segundo pesquisa realizada pelos Conselhos Federal de Medicina (CFM) e Regional de Medicina do Estado de São Paulo (CREMESP), havia, no final de 2011, 371.788 médicos em atividade no Brasil. Nos últimos 40 anos, teria havido crescimento desse número da ordem de $530 \%$, muito superior ao da população brasileira, que teria sido de 105\% no mesmo período (CFM/CREMESP, 2011, p. 15). Porém, a distribuição desses profissionais pelas diferentes regiões do país é bastante desigual: enquanto o número de médicos por mil habitantes no país é de 1,95, na Região Norte é de apenas 0,99 e na Região Sudeste é de 2,61. A Região Nordeste também tem um índice baixo, de apenas 1,19 médicos por mil habitantes (CFM/CREMESP, 2011, p. 29). Apenas 55\% dos médicos brasileiros teriam título de especialistas obtidos junto às sociedades de especialidades ou programas de residência médica (p. 55). O Programa Mais Médicos pretende enfrentar tais problemas ampliando as vagas de graduação e residência médica, principalmente nas áreas mais carentes desses profissionais.

Como mencionamos anteriormente, alguns países buscam regular o trabalho médico através do controle de estoque e a formação para as especialidades. No nosso país, no entanto, não há tradição de "regulação pelo Estado do exercício profissional, da formação, da certificação de competência ou da autorização de práticas de trabalho" (CECCIM; PINTO 2007, p. 269). A Constituição Federal estabeleceu entre as competências do SUS o ordenamento da formação de 
recursos humanos na área de saúde (Art. 200, III). Seria de se esperar que, com o advento do SUS, o Estado procurasse intervir sobre o estoque de profissionais, no sentido de promover a formação de especialistas de acordo com as necessidades da população e a expansão da oferta de serviços públicos.

Uma das poucas iniciativas estatais que pode ser associada a um ordenamento da formação de médicos foi a publicação, pelo Ministério da Educação, das novas Diretrizes Curriculares Nacionais do Curso de Graduação em Medicina (BRASIL, 2001), que procuraram fortalecer o conteúdo de saúde pública. Só a partir de 2003, com a criação da Secretaria de Gestão do Trabalho e da Educação na Saúde (SGTES), o Ministério da Saúde começou a propor as primeiras políticas nesse sentido, ainda sem resultados significativos.

A formação para as especialidades médicas, através de programas de residência, foi instituída no Brasil pelo Decreto no 80.281/77 (BRASIL, 1977), mais de dez anos antes da criação do SUS. O decreto criou, ainda, uma Comissão Nacional de Residência Médica (CNRM), responsável pela coordenação das iniciativas na área e pelo credenciamento dos programas (Art. $2^{\circ}$ ). Metade dos dez integrantes da mesma era composta por representantes diretos ou indiretos da categoria médica - Conselho Federal de Medicina; Associação Brasileira de Escolas Médicas; Associação Médica Brasileira; Federação Nacional dos Médicos; e Associação Nacional de Médicos Residentes (art. Art. $2^{\circ}, \mathbb{\$} 1^{\circ}$ ). O decreto estabeleceu ainda que os programas devessem ser desenvolvidos, preferencialmente, nas áreas de Clínica Médica; Cirurgia Geral; Pediatria; Obstetrícia e Ginecologia; e Medicina Preventiva ou Social (Art. $1^{\circ}, \$ 1^{\circ}$ ), favorável, portanto, à formação de médicos para a atenção básica ou primária. Vale lembrar que a Medicina Geral e Comunitária, depois Medicina de Família e Comunidade, já estava regulamentada pela CNRM, mas não entrou neste rol de especialidades básicas.

Em 2011, foi sancionado o Decreto ${ }^{\circ}$. 7.562, que estabeleceu que:

\begin{abstract}
A regulação das instituiçôes e dos programas de residência médica deverá considerar a necessidade de médicos especialistas indicada pelo perfil socioepidemiológico da população, em consonância com os princípios e as diretrizes do Sistema Único de Saúde - SUS (Art. 2º, parágrafo único).
\end{abstract}

Segundo o mesmo documento, essa função deverá ser "exercida por meio da expedição de atos autorizativos para o funcionamento de instituições e de programas de residência médica" (Art. 14), porém sem nenhuma recomendação ou forma de operacionalização na intenção de considerar as necessidades de especialistas 
por parte do SUS. Somente com a publicação da Lei no $12.871 / 2013$, o Estado procurou considerar tais necessidades, mas de forma ainda um tanto vaga, pois não foi instituída uma relação numérica entre demanda e oferta desses profissionais.

O decreto também alterou a composição da CNRM, que passou a contar com representantes do Conselho Nacional de Secretários de Saúde (CONASS) e do Conselho Nacional de Secretários Municipais de Saúde (CONASEMS). A representação da categoria médica foi ampliada de cinco para sete membros: um representante da Federação Brasileira de Academias de Medicina (FBAM); e "um médico de reputação ilibada, docente em cargo de provimento efetivo em Instituição de Educação Superior pública [...]” (Art. 4º, X e XI). Resta saber se esta composição da Comissão favorece ao propósito de regulação dos programas em favor do SUS estabelecido no parágrafo único do artigo $2^{\circ}$, uma vez que a categoria médica conta com sete representantes, e o SUS, com apenas três Ministério da Saúde, CONASS e CONASEMS.

Ainda que não haja controle de estoque de médicos, nem planejamento de sua distribuição com base nas necessidades de saúde, houve uma iniciativa de avaliar as necessidades de médicos especialistas em 2007, por parte dos Ministérios da Educação e da Saúde (RIBEIRO, 2011). O relatório resultante desse estudo, no entanto, apenas definiu como prioridades as áreas "Atenção Básica, Saúde Mental, Atenção Oncológica, Atenção Obstétrica e Pediátrica e Urgências/ Emergências", não enfatizando a especialidade de Medicina de Família e Comunidade (BRASIL, 2009, p. 5). O relatório tampouco apresentou números concretos em relação a cálculos de necessidades, concluindo com a sugestão de realização "em cada Estado [de] um Plano de ação estadual que [...] deverá conter as necessidades de médicos especialistas em cada Estado, bem como a capacidade instalada para abertura de novos programas" (BRASIL, 2009, p. 10).

Feuerwerker (1998, p. 62) destaca outro fator importante sobre a regulação das especializações médicas no nosso país: "A Residência Médica no Brasil [...] tem tido seus rumos definidos [...] em uma relação mediatizada pelos interesses da corporação médica: médicos-residentes, sociedades especialistas e outras entidades". É como se existisse, desde 1977, um acordo tácito entre o Estado (CNRM) e as entidades corporativas médicas em torno da conciliação de interesses, de ambos os lados, ao serem elevados os programas oficiais de residência à categoria de título de especialista, permanecendo válidos os títulos 
conferidos pelas sociedades médicas. Uma evidência deste acordo tácito ocorreu em 1989, apenas um ano após a criação do SUS, ao ser firmado convênio entre o Conselho Federal de Medicina (CFM) e a Associação Médica Brasileira (AMB), pelo qual as duas entidades resolveram só reconhecer os especialistas que se submetam aos exames das sociedades médicas especializadas, sem que a CNRM tenha esboçado qualquer reação (RIBEIRO 2011, p. 6).

No início de 2002, esse acordo tácito foi formalizado por meio de convênio firmado entre a CNRM, a AMB e o CFM, e ratificado em Resolução do CFM (RIBEIRO, 2011, p. 6-7). Por conta desse convênio, passou a caber à AMB "orientar as suas sociedades de especialidade e fiscalizar a forma de concessão de títulos e certificados emitidos pelas mesmas em conformidade com este convênio" (CFM, 2002, Cláusula Primeira). O acordo e o convênio colocaram nas mãos da própria corporação médica uma influência decisiva sobre a formação profissional, exatamente quando se implantava a Reforma Sanitária e havia necessidade de um reordenamento desses profissionais nos estabelecimentos de saúde em expansão. O recente Decreto no 7.562/2011 é omisso a este respeito, mantendo, portanto, a influência da corporação médica sobre a formação e regulação dos especialistas no mercado de trabalho.

\section{Formação para a especialidade}

Segundo Gusso (17/10/11), a especialidade de Medicina de Família e Comunidade no Brasil existe desde 1976, mas só foi reconhecida pela CNRM em 1981, como Medicina Geral Comunitária, passando a ser denominada com o nome atual em 2001. Da mesma forma, Anderson e Rodrigues (2008, p. 30) confirmam o início da formação para a especialidade em 1976, quando foram criados programas de residência médica pela Universidade do Estado do Rio de Janeiro (UERJ), pela Universidade Federal de Pernambuco (UFPE) e pela Escola de Saúde Pública da Secretaria de Estado de Saúde do Rio Grande do Sul. Na UERJ, tratava-se de um programa de formação em Medicina Integral e nos dois últimos recebiam a denominação de Medicina Geral Comunitária.

A criação, em 1994, do Programa Saúde da Família (PSF), não foi acompanhada pela oferta de vagas de residência em Medicina de Família e Comunidade. Na falta de especialistas, qualquer médico poderia trabalhar no programa, mesmo 
carecendo de formação adequada. Com a expansão do programa, a partir de 1999 (BRASIL, MS/DAB, acesso em 20/08/12), a necessidade de formação de especialistas se tornou ainda mais premente. Só em 2005, entretanto, o Ministério da Saúde passou a apoiar a formação de especialistas através de programas de residência (BRASIL, 2005). Em 2009, foi instituído o Programa Nacional de Apoio à Formação de Médicos Especialistas em Áreas Estratégicas - PRÓ-RESIDÊNCIA (Portaria interministerial MEC/MS no 1.001/2009), visando favorecer a formação de especialistas em áreas prioritárias, oferecendo 788 vagas para cinco especialidades básicas e prioritárias, entre as quais se incluía a Medicina de Família e Comunidade (RIBEIRO, 2011, p. 34). O último dado disponível em relação ao número de vagas para residência em Medicina de Família e Comunidade é de 2009, totalizando apenas 584 vagas para ingresso nos 85 programas existentes em todo o país (BRASIL, CNRM, acesso em 09/08/12).

Como apontam Anderson et al. (2007), a necessidade de expansão da Residência Médica para a especialidade necessitaria ser apoiada pela formação de um número adequado de preceptores. $\mathrm{O}$ fato de não haver número suficiente de médicos de família e comunidade com titulação por programa de residência constitui um obstáculo para o crescimento da oferta de vagas para residentes.

As iniciativas de expansão e incentivo da residência no país, além de recentes, ainda parecem tímidas, como é o caso do Programa Nacional de Apoio à Formação de Médicos Especialistas em áreas estratégicas - PRÓ-RESIDÊNCIA (Portaria Interministerial $n^{\circ}$ 1001/2009). Este se propõe a ampliar o número de vagas em residência em regiôes prioritárias do país, novamente sem qualquer referência a números relacionados com oferta e demanda, nem trata da residência em medicina de família e comunidade. O Programa Mais Médicos tampouco procura relacionar a quantidade de vagas de residência em função do conhecimento dos números de oferta e demanda. Estabelece, entretanto, uma série de exigências novas em relação ao total de vagas (que deve ser equivalente ao de egressos de em medicina em cada ano, até 2018), prevê o acesso direto à residência em algumas especialidades e exige que o primeiro ano do Programa de Residência em Medicina Geral de Família e Comunidade seja obrigatório para o ingresso para determinados programas de residência (Medicina Interna (Clínica Médica); Pediatria; Ginecologia e Obstetrícia; Cirurgia Geral; Psiquiatria; e Medicina Preventiva e Social). 
Deve-se lembrar que não há, no Brasil, exigência de conclusão de programa de residência médica para o exercício da profissão no sistema público, nem no privado. Para trabalhar como especialista, basta o profissional fazer algum curso de especialização ou se submeter a uma prova de titulação de alguma sociedade médica especializada. Esta situação, por si só, demonstra a importância das sociedades especializadas na formação profissional no país. Como não há qualquer regulação para o ingresso nos programas de residência, baseado em controle público sobre o estoque de profissionais, os recém-formados escolhem livremente cursar ou não uma residência médica. No caso da Saúde da Família, especificamente, não há sequer diferenciação instituída pelo governo em termos de remuneração entre os profissionais formados para a especialidade e os que não o são, o que contribui para reduzir a atratividade dos programas de residência.

É interessante fazer algumas comparações internacionais neste ponto para ilustrar a debilidade dos mecanismos de formação de médicos de família. Segundo Heyrman et al. (2006, p. 245-246), a Medicina da Família e Comunidade tem papel central no ensino de graduação na Europa. Desde 2001, na União Europeia, a duração mínima da residência em Medicina de Família e Comunidade é de três anos, enquanto no Brasil esse período é de apenas dois anos. Na Espanha, onde o tempo mínimo de residência é quatro anos (ESPANHA, 2005), um Decreto em 1984 determinou que o número de vagas oferecidas para todas as especialidades médicas fosse fixado pelos Ministérios de Educação e de Saúde, com base nas necessidades de saúde, e que um único exame para todas as especialidades fosse realizado de forma centralizada pelo Estado. Outra diferença nítida entre as normas espanholas e as brasileiras sobre a formação especializada de médicos é que lá as corporaçôes médicas não influenciam diretamente o processo de formação, cujo controle é do Estado (ESPANHA, 1984, p. 5.1, 2, A, B e C).

Pode-se dizer, em suma, que o Brasil ainda carece de maior oferta de vagas de residência em Medicina de Família e Comunidade, assim como de incentivos para atrair profissionais para a especialidade. $\mathrm{Na}$ ausência de mecanismos de regulação da oferta de profissionais médicos e de políticas de estímulo efetivas para sua formação especializada e permanência no PSF, é razoável supor que os interesses do mercado continuem a prevalecer e que o SUS continuará a ter problemas para atrair e manter médicos de família na ESF. Há um aparente descompasso entre a retórica oficial, que atribui grande importância à Saúde da Família e as medidas efetivas de formação e regulação da especialidade no país. 


\section{Regulação do trabalho na Estratégia Saúde da Família}

Desde o início da implantação da Saúde da Família, as normas do Ministério da Saúde foram vagas a respeito do trabalho médico. Uma das primeiras normas específicas para regulamentar esse trabalho no Programa Saúde da Família foi a Portaria GM/MS no 1.886/1997, que considera a presença do médico como obrigatória para a constituição das Equipes de Saúde da Família, mas não estabeleceu responsabilidades desses profissionais (BRASIL, MS/GM, 1997, Anexo 2, item 11). No mesmo ano, documento de política da Secretaria de Assistência à Saúde do Ministério, intitulado "Saúde da Família - uma estratégia de reorientação do modelo assistencial”, preconizava que o médico da equipe deveria ser "preferencialmente [...] um generalista" (BRASIL, 1997, p. 15-16). Os "Princípios e Diretrizes para a NOB/RH-SUS", estabelecidos pela Conferência Nacional de Saúde, na sua única referência à Saúde da Família estabelecia a necessidade de "priorizar a capacitação de recursos humanos para os programas de saúde da família” (BRASIL, 2005, p. 59).

A partir da introdução da Política Nacional de Atenção Básica (Portaria GM/ MS no. 648/2006), pela primeira vez a gestão do trabalho dos profissionais inseridos na atenção primária no país passou a contar com diretrizes mais claras. A política caracterizava um conjunto de ações, em âmbito individual e coletivo, como parte das iniciativas de organização da atenção básica tanto para o PSF, quanto para o PACS, definindo as responsabilidades das três esferas de governo, as atribuiçõos de cada membro das ESFs e a infraestrutura de recursos necessários para sua implantação. No que tange à jornada de trabalho dos seus profissionais, a portaria definiu:

[...] jornada de trabalho de 40 horas semanais para todos os seus integrantes e composta por, no mínimo, médico, enfermeiro, auxiliar de enfermagem ou técnico de enfermagem e Agentes Comunitários de Saúde. (BRASIL, 2006, item 3, I).

Essa portaria representou um inegável avanço na regulação dos processos de trabalho no âmbito da atenção primária brasileira. Apontava a necessidade de trabalho em equipes multiprofissionais, responsáveis pela prestação de serviços em determinada área de abrangência na qual os profissionais deveriam estabelecer vínculos mais permanentes de confiança com os usuários, centrada na determinação social da doença (HEIMANN; MENDONÇA, 2005). O regime de trabalho de 40 horas semanais para todos os profissionais e o trabalho em equipe eram 
premissas básicas da proposta. Durante todo o processo de desenvolvimento da estratégia, entretanto, uma das maiores dificuldades até os dias atuais é a atração e manutenção dos médicos nas equipes, como já mencionamos.

Em 2011, o Ministério da Saúde, na tentativa de atrair mais médicos para a Saúde da Família, resolveu flexibilizar a carga horária dos profissionais médicos das ESFs, através da nova Política Nacional de Atenção Básica (Portaria GM/ MS no. 2.488/2011). A referida portaria também modificou a forma de repasse do Piso de Atenção Básica (PAB Variável), de acordo com os diferentes regimes de trabalho dos médicos, ou "configurações das equipes". Estas passaram a ter número e carga de trabalho médico variado, mantendo inalterados, no entanto, o quantitativo e a carga de trabalho das demais categorias profissionais que as compõem. As alteraçôes introduzidas são sintetizadas na tabela 1, a seguir.

Tabela 1. Novas configurações de Equipes de Saúde da Família, carga horária dos médicos e respectivo incentivo do PAB Variável

\begin{tabular}{l|r|r|r|r}
\hline Configurações & $\begin{array}{c}\text { No de ESF ou } \\
\text { equivalente } \\
\text { contingente } \\
\text { populacional }\end{array}$ & No de médicos & $\begin{array}{c}\text { Carga horária } \\
\text { semanal (h) }\end{array}$ & $\begin{array}{c}\text { PAB Variável } \\
(\%)\end{array}$ \\
\hline original & 1 & 1 & 40 & 100 \\
\hline I & 1 & 2 & 30 & 100 \\
\hline II & 2 & 3 & 30 & 100 \\
\hline III & 3 & 4 & 30 & 100 \\
\hline IV & 1 & 2 & 20 & 85 \\
\hline V $\left.*^{*}\right)$ & 1 & 1 & 20 & 60 \\
\hline
\end{tabular}

Fonte: Elaboração própria, a partir da Portaria GM/MS no. 2.488/2011.

(*) Equipe transitória, cujo número varia em função da população municipal.

Além da configuração original, oriunda da implantação do programa, a portaria possibilitou cinco modalidades de equipe, cujos médicos possuem cargas horárias de 20, 30 ou 40 horas semanais. Não especifica como tais cargas horárias devem ser distribuídas ao longo da semana, o que permite criar uma grande variação nos processos de trabalho. Essa flexibilização abre a possibilidade de conflitos com os outros profissionais, uma vez que só se aplica à categoria 
médica. A integração entre os profissionais também pode ficar comprometida, pois em cada turno pode estar presente um médico diferente. E por fim, pode interferir nos cuidados e nos vínculos entre médico e paciente, uma vez que passa a ser possível que mais de um médico acompanhe o mesmo paciente.

Fontenelle (2012, p. 6) chama a atenção, ainda, para a viabilidade do cadastro de equipes em caráter transitório, com apenas um médico com carga horária de 20 horas, sem que se estabeleça um prazo para tal transitoriedade. O prazo fica a critério do gestor, havendo apenas a recomendação de que este "tão logo tenha condiçôes, transite para os formatos anteriores", o que parece bastante vago. A falta de critérios para a distribuição da carga horária pode prejudicar o processo de trabalho da equipe, ao possibilitar que não haja médico em alguns horários de funcionamento das unidades, prejudicando principalmente os casos de urgência e de longitudinalidade do cuidado.

Esta multiplicidade de possibilidades pode representar um retrocesso quanto ao funcionamento das equipes e à regulação do trabalho na Saúde da Família e $\mathrm{o}$ abandono de uma regra de isonomia entre os profissionais que as compõem. A medida recente equivale, portanto, a uma redução da regulação estatal sobre o exercício do trabalho médico na estratégia.

\section{Considerações finais}

O setor de saúde brasileiro tem uma trajetória de déficit regulatório em matéria de gestão da formação profissional e do exercício do trabalho. Isso tem implicações negativas em relação à gestão do sistema e à qualidade da assistência. Esse déficit resulta em estímulos indiretos aos interesses privados e corporativos, e contrasta com o que ocorre em algumas experiências internacionais exitosas no campo da atenção primária.

Pode-se considerar que há contradição entre as medidas tomadas em 2011 e em 2013. Enquanto a primeira flexibilizou a carga horária dos médicos na Saúde da Família, a segunda pretende criar condições para oferecer mais profissionais para a atenção básica, principalmente em áreas mais carentes, além de regular a formação de especialistas em função das necessidades de saúde e dos princípios do SUS. Cabe perguntar qual incentivo existiria para a formação na especialidade, quando o exercício profissional na área equivalente passou a poder ser exercido em horário parcial, para o qual sequer se exige a formação específica. 
Ao assumir uma posição titubeante, entre a regulação e a desregulação, o Estado brasileiro pode ter optado por ceder ante aos interesses do mercado e da corporação médica. A intenção de "regular" a formação deveria estar acompanhada de medida equivalente em relação ao exercício da profissão. Tratase de uma opção oposta a de outros países, que enfatizam a importância da atenção primária e a filosofia da saúde da família como lógica de trabalho.

A opção adotada equivale a colocar todas as fichas na geração de "estímulos" e facilidades para a inserção dos médicos na Saúde da Família, relegando a um nível mínimo o papel regulador do Estado quanto à formação e o exercício profissional. É como se houvesse a esperança, que nos parece infundada, de que uma baixa intervenção regulatória representasse um atrativo para a estratégia. Essa opção parece abstrair o fato de que há uma série de estímulos muito mais fortes por parte dos interesses de mercado do que uma flexibilidade de horários. ${ }^{4}$

\section{Referências}

ANDERSON, M.I.P.; RODRIGUES, R.D. Formação em Medicina de Família e Comunidade. Cadernos ABEM, Florianópolis, v. 6, n. 18, p. 19-20, 2008.

ANDERSON, M.I.P. et al. Bases para expansão e desenvolvimento adequados de programas de Residência em Medicina de Família e Comunidade. Rev Bras Med Fam e Com., Rio de Janeiro, v. 3, n. 11, p. 180-198, 2007.

BERMAN, S. The primacy of politics: Socialdemocracy and the making of Europe's twentieth century. New York: CambridgeUniversity Press, 2006. 228p.

BLOOR, K.; MAYNARD, A Planning human resources in health care: Towards an economic approach, an international comparative review. Disponível em: <http://www.chsrf.ca/ PublicationsAndResources/ResearchReports/CommissionedResearch/03-03-01/a8ae7a7e79f3-47f5-9f65-92b9fe71b873.aspx>. Acesso em: 20 fev. 2011. 27p.

BOERMA, W.G.W.; RICO, A. Alteração das condições para a reforma estrutural na atenção primária. In: SALTMAN, R.B.; BOERMA, W.G.W.; RICO, A (eds.). Atenção primária conduzindo as redes de atenção à saúde: reforma organizacional na atenção primária europeia. Berkshire: Open University Press, 2006. p. 89-111.

BRASIL. Ministério da Educação. Portaria Interministerial no .1 .001 , de 22 de outubro de 2009. Institui o Programa Nacional de Apoio à Formação de Médicos Especialistas em Áreas Estratégicas - PRÓ-RESIDÊNCIA. Diário Oficial [da] República Federativa do Brasil, Brasília, DF, n. 203, Seção I, p. 9, out. 2009. 
BRASIL. Ministério da Educação. Conselho Nacional de Educação. Câmara de Educação Superior. Resolução CNE/CES no. 4, de 7 de novembro de 2001. Institui Diretrizes Curriculares Nacionais do Curso de Graduação em Medicina. Diário Oficial [da] República Federativa do Brasil, Brasília, DF, v. 0, n. 0, Seção I, p. 38,nov.2001.

. Sistema da Comissão Nacional de Residência Médica. Instituiçôes x Programas $x$ Vagas. Disponível em: <http://mecsrv04.mec.gov.br/sesu/SIST_CNRM/APPS/inst_ especialidades.asp >. Acesso em: 09 ago. 2012.

BRASIL. Ministério da Educação.Ministério da Saúde. Comissão Interministerial de Gestão da Educação na Saúde. Avaliação das necessidades de médicos especialistas. Relatório produzido pela Subcomissão de Estudo e Avaliação das Necessidades de Médicos Especialistas no Brasil. Brasília: MEC/MS, 2009.10p.

BRASIL. Ministério da Saúde. Portaria Interministerial no 1,369, de 8 de julho de 2013. Dispõe sobre a implementação do Projeto Mais Médicos. Diário Oficial [da] República Federativa do Brasil, Brasília, DF, n. 219, Seção 3, p. 204 nov. 2013.

BRASIL. Ministério da Saúde. Ministério da Educação. Portaria Interministerial no 2.101, de 3 de novembro de 2005. Programa Nacional de Reorientação da Formação Profissional em Saúde - PRÓ-SAÚDE. Diário Oficial [da] República Federativa do Brasil, Brasília, DF, v. 0, n. 0, Seção I, p. 111, nov. 2005.

BRASIL. Ministério da Saúde. Fundo Nacional de Saúde. Disponível em: <http://www. fns.saude.gov.br/Prog_PAB-FIXO.asp>. Acesso em: 18 nov. 2011.

BRASIL. Ministério da Saúde. Secretaria de Assistência à Saúde. Coordenação de Saúde da Comunidade. Saúde da Família: uma estratégia para a reorientação do modelo assistencial. Brasília: Ministério da Saúde, 1997. 36p.

Programa Saúde da Família. Disponível em: <http://portal.saude.gov.br/portal/ saude/cidadao/area.cfm?id_area=149>. Acesso em: 07 nov. 2011.

BRASIL. Ministério da Saúde. Conferência Nacional de Saúde. Documentos preparatórios para a Terceira Conferência Nacional de Gestão do Trabalho e da Educação na Saúde. Brasília: Ministério da Saúde/Conselho Nacional de Saúde, 2005. 73p.

BRASIL. Ministério da Saúde e Conselho Nacional de Saúde. Princípios e Diretrizes para a NOB/RH-SUS. Brasília: Ministério da Saúde, 2003. 112p (Série Cadernos Técnicos, Série J. Cadernos).

BRASIL. Ministério da Saúde, Gabinete do Ministro. Portaria GM/MS no. 2.488, de 21 de outubro de 2011. Aprova a política nacional de atenção Básica, estabelecendo a revisão de diretrizes e normas para a organização da atenção básica, para a Estratégia Saúde da Família (ESF) e o Programa de Agentes Comunitários de Saúde (PACS). Diário Oficial [da] República Federativa do Brasil, Brasília, DF, v. 0, n. 0, Seção I, p. 48, out. 2011. 
Portaria GM/MS No 3.839, de 7 de dezembro de 2010. Estabelece incentivo para equipes de Saúde da Família que contem com profissionais certificados para atuação na Atenção Primária à Saúde.Diário Oficial [da] República Federativa do Brasil, Brasília, DF, v. 0, n. 0, p. 44, dez. 2010.

. Portaria GM/MS no. 648, de 28 de março de 2006. Aprova a Política Nacional de Atenção Básica, estabelecendo a revisão de diretrizes e normas para a organização da Atenção Básica para o Programa Saúde da Família (PSF) e o Programa Agentes Comunitários de Saúde (PACS). Diário Oficial [da] República Federativa do Brasil, Brasília, DF, v. 0, n. 0, Seção I, p. 71, mar. 2006.

Portaria GM/MS no. 1.143, de 7 de julho de 2005. Apoia programas de residência médica em medicina de família e comunidade (PRM-MFC), por meio do Programa de Bolsas para a Educação pelo Trabalho, do Ministério da Saúde. Diário Oficial [da] República Federativa do Brasil, Brasília, DF, v. 0, n. 0, p. 30, jul. 2005. Seção I. Diário Oficial [da] República Federativa do Brasil, Brasília, DF, v. 0, n. 0, Seção I, p. 30, jul. 2005.

Portaria GM/MS no 1886, 18 de dezembro de 1997. Aprova as Normas e Diretrizes do Programa de Agentes Comunitários de Saúde e do Programa de Saúde da Família. Diário Oficial [da] República Federativa do Brasil, Brasília, DF, v. 0, n. 0, Seção I, p. 247, dez. 1997. BRASIL. Ministério da Saúde. Secretaria de Políticas de Saúde, Departamento de Atenção Básica. Avaliação da implementação do Programa de Saúde da Família em dez grandes centros urbanos: síntese dos principais resultados. Brasília: Ministério da Saúde, 2002. 210p.

. Teto, credenciamento e implantação das estratégias de Agentes Comunitários de Saúde, Saúde da Família e Saúde Bucal Unidade Geográfica: Brasil Competência: junho de 2012 a julho de 2012. Disponível em: <http://dab.saude.gov.br/historico_cobertura_sf.php>. Acesso em: 09 ago. 2012.

. Meta e evolução do número de Equipes de Saúde da Família implantadas, Brasil 1994-agosto 2011. Disponível em: <http://dab.saude.gov.br/abnumeros.php\#mapas>. Acesso em: 20 ago. 2012.

BRASIL. Presidência da República. Decreto no 80.281, de 5 de setembro de 1977. Regulamenta a Residência Médica, cria a Comissão Nacional de Residência Médica e dá outras providências. Diário Oficial [da] República Federativa do Brasil, Brasília, DF, v. 0, n. 0, Seção I, p. 11.787, set. 1977.

. Decreto no. 7.562, de 15 de setembro de 2011. Dispõe sobre a Comissão Nacional de Residência Médica e o exercício das funções de regulação, supervisão e avaliação de instituições que ofertam residência médica e de programas de residência médica. Diário Oficial [da] República Federativa do Brasil, Brasília, DF, v. 0, n. 0, Seção I, p. 3, set. 2011.

CECCIM, R.B.; PINTO, L.F. A formação e especialização de profissionais de saúde e a necessidade política de enfrentar as desigualdades sociais e regionais. Revista Brasileira de Educação Médica, Rio de Janeiro, v. 29, n. 1, p. 266-277, 2007. 
CONSELHO FEDERAL DE MEDICINA. Resolução nº 1.634, de 11 de abril de 2002.

Dispõe sobre convênio de reconhecimento de especialidades médicas firmado entre o Conselho Federal de Medicina CFM, a Associação Médica Brasileira - AMB e a Comissão Nacional de Residência Médica - CNRM. Diário Oficial [da] República Federativa do Brasil, Brasília, DF, v. 0, n. 0, Seção I, p. 81, abr. 2002.

CONSELHO REGIONAL DE MEDICINA DO ESTADO DE SÃO PAULO. CONSELHO FEDERAL DE MEDICINA. Demografia Médica no Brasil: dados gerais e descrições de desigualdades. São Paulo: CREMESP-CFM, 2011.118p.

DEPARTAMENTO DE INFORMÁTICA DO SUS. CNES - Equipes de Saúde - Brasil, Equipes de Saúde da Família. Disponível em:<http://tabnet.datasus.gov.br/cgi/tabcgi. exe?cnes/cnv/equipebr.def>. Acesso em: 04 mar. 2011.

ELEY, G. Forjando a democracia, a história da esquerda na Europa, 1850-2000. São Paulo: Fundação Perseu Abramo, 2005. 768p.

ESCOREL, S.; GIOVANELLA, L.; MENDONÇA, M.H.; SENNA, M.C.M. O Programa de Saúde da Família e a construção de um novo modelo para a atenção básica no Brasil. Rev Panam Salud Publica, Washington v. 21, n.2, p.164-176, 2007.

ESPANHA. Real Decreto 127/1984, de 11 de enero, por el que se regula la formación médica especializada y la obtención del título de Médico Especialista. Boletín Oficial de España, p. 2524, Ene. 1984 (BOE-A-1984-2426).

ESPANHA. Comisión Nacional de la Especialidad de Medicina Familiar y Comunitaria. Ministerio de Sanidad y Consumo. Ministerio de Educación y Ciencia. Programa Formativo de la Especialidad de Medicina Familiar y Comunitaria. Madrid: Comisión Nacional de la Especialidad de Medicina Familiar y Comunitaria, 2005. 136p.

FELDSTEIN, P.J. Health Care Economics. New York: Wiley Medical Publication, 1998. 541 p. FEUERWERKER, L.C.M. Mudanças na educação médica e residência médica no Brasil. Interface-Comunic, Saúde, Educ. Botucatu, v. 2, n. 3, p .51-71, 1998.

- Modelos tecnoassistenciais, gestão e organização do trabalho em saúde: nada é indiferente no processo de luta para a consolidação do SUS. Interface: Comunic, Saúde, Educ, Botucatu, v. 9, n. 18, p. 489-506, 2005.

FONTENELLE, L.F. Mudanças recentes na Política Nacional de Atenção Básica: uma análise crítica. Rev bras med fam comunidade. Florianópolis, v. 7, n. 22, 2012 (no prelo).

FREIDSON, E. Profissão médica: um estudo de sociologia do conhecimento aplicado. São Paulo: UNESP, 2009. 453 p.

GIL, C.R.R. Atenção primária, atenção básica e saúde da família: sinergias e singularidades do contexto brasileiro. Cad. Saúde Pública. Rio de Janeiro, v. 22, n. 6, p. 1171-1181, 2006.

GIOVANELLA, L. A atenção primária à saúde nos países da União Europeia: configurações e reformas organizacionais na década de 1990. Cad. Saúde Pública. Rio de Janeiro, v. 22, n. 5, p. 951-963, 2006. 
GIRARDI, S.N.; CARVALHO, C.L. Configuraçôes do mercado de trabalho dos assalariados em saúde no Brasil. Disponível em: www.opas.org.br/rh/admin/documentos/mtlast.PDF. Acesso em: 15 jun. 2010.

GIRARDI, S.N.; CARVALHO, C.L. Contratação e qualidade do emprego no Programa de Saúde da Família no Brasil. In: FALCÃO, A.R.B. et al. (Orgs.). Observatório de recursos humanos no Brasil: estudos e análises. Rio de Janeiro: Fiocruz, 2003. p. 157-190.

GUSSO, G. A especialidade Medicina de Família e Comunidade. Disponível em: http:// www.apmfc.org.br/App_Files/Artigos/especialidade.pdf. Acesso em: 17 out. 2011.

HEIMANN, L.S.; MENDONÇA, M.H. A trajetória da Atenção Básica em saúde e do Programa de Saúde da Família no SUS: uma busca de identidade. In: LIMA, N. et al. (Orgs.). Saúde e democracia: história e perspectivas do SUS. Rio de Janeiro: Fiocruz, 2005. p. 481-502.

JUDT, T. Pós Guerra: uma história da Europa desde 1945. Rio de Janeiro: Objetiva, 2008. 996p.

HEYRMAN, J. et al. Mudanças nos papéis profissionais na formação em atenção primária. In: SALTMAN, R.B.; BOERMA, W.G.W.; RICO, A. (eds.). Atenção primária conduzindo as redes de atenção à saúde: reforma organizacional na atenção primária europeia. Berkshire: Open University Press, 2006. p. 237-261.

HOBSBAWN, E.J. A era dos extremos: o breve século XX: 1914-1991. São Paulo: Cia das Letras, 1995.608 p.

IUNES, R.F. Demanda e demanda em saúde. In: PIOLA, S.F.; VIANNA, S.M. (orgs.). Economia da Saúde: conceito e contribuição para a gestão da saúde. Brasília: IPEA, 1995. p. 99-140.

LIMONCIC, F. Os inventores do New Deal: Estado e sindicatos no combate à Grande Depressão. Rio de Janeiro: Civilização Brasileira, 2009. 288P.

MINTZBERG, Henry. Criando organizaçôes eficazes, estruturas em cinco configurações. São Paulo: Atlas, 1995. 304 p.

MENELEU NETO, J. et al. O trabalho do médico: de profissional liberal a assalariado. Fortaleza: ROREHS-CETREDE/UFC/UECE, 2006. 39p.

NEY, M.S. Condições de fixação do médico no Programa Saúde da Família. 2009. 122p. Dissertação (Mestrado em Saúde da Família) - Mestrado em Saúde da Família, Universidade Estácio de Sá, Rio de Janeiro, 2009.

ORGANISATION FOR ECONOMIC CO-OPERATION AND DEVELOPMENT. OECD Health Data 2011, Physicians by category. Disponível em: <http://stats.oecd.org/ index.aspx?DataSetCode=HEALTH_STAT>. Acesso em: 05 set. 2011.

POLANYI, K. The great transformation, the political and economic origins of our time. Boston: Beacon Press, 1998. 317p. 
(Mestrado em Saúde da Família) - Mestrado em Saúde da Família, Universidade Estácio de Sá, Rio de Janeiro, 2011.

RIBEIRO, M.A.A. Apontamentos sobre residência médica no Brasil. Brasília: Câmara dos Deputados, 2011. 69p.

SAYEG, M.A. Correlaçôes numéricas entre médicos e outros profissionais de nível superior. Revista de Administração Pública. Rio de Janeiro, v. 11, n. 3: 111-131, jul-set, 1977.

SCHWEITZER, S. O. Pharmaceuticals economy and policy. New York: Oxford University Press, 2007. 413p.

STARR, P. The Social Transformation of American Medicine: The rise of a sovereign profession and the making of a vast industry. New York: Perseus Books, 1982. 514 p.

VIANA, A.L.D.; DAL POZ, M.R. A Reforma do Sistema de Saúde no Brasil e o Programa de Saúde da Família. Physis: Revista de Saúde Coletiva. Rio de Janeiro, v. 15 (supl.), p. $225-$ $264,2005$.

WILLIS, E. Medical dominance: The division of labour in Australian health care. Sidney and Boston: Allen \& Unwin, 1983, 235 p.

WONG, L.R. et al. Estimativas de mão de obra qualificada para o curto e médio prazo: uma proposta metodológica aplicada ao caso das especialidades médicas em Minas Gerais (Trabalho submetido à consideração do XV Seminário sobre a Economia Mineira organizado pelo Cedeplar (2012). Disponível em: <http://web.cedeplar.ufmg.br/cedeplar/ seminarios/ecn/ecn-mineira/2012/arquivos/Estimativas\%20de\%20m\%C3\%A3o\%20 de\%20obra\%20qualificada\%20para\%20o\%20curto\%20e\%20m\%C3\%A9dio\%20prazo. pdf>. Acesso em: 19 out 2012.

\section{Notas}

${ }^{1}$ MS/SAS/Departamento de Atenção Básica - DAB Teto, credenciamento e implantação das estratégias de Agentes Comunitários de Saúde, Saúde da Família e Saúde Bucal Unidade Geográfica: Brasil Competência: Junho de 2012 a Julho de 2012 (acesso em 09/08/12).

${ }^{2} \mathrm{O}$ termo estoque (de profissionais médicos) vem do inglês stock of doctors (BLOOR; MAYNARD, 2003), e vem sendo utilizado na literatura sobre recursos humanos em saúde no Brasil pelo menos desde 1977. Ver, por exemplo, os trabalhos de Sayeg (1977) e de Wong et al. (2012).

${ }^{3}$ Quando o artigo foi submetido, o programa ainda não existia. Como o tema tratado tem relação direta com o mesmo, os autores solicitaram a inclusão de referência ao mesmo ao editor.

${ }^{4}$ P.H.A. Rodrigues e M.S. Ney participaram da concepção do artigo, análise da literatura e normas existentes, escrita da versão final e revisão do artigo. C.H.A. Paiva e L.M.B.M. Souza participaram da concepção do artigo, escrita da primeira versão e revisão do artigo. 
Regulation of medical labor in Brazil: impacts on Family Health Care Strategy

The article reviews labor regulation of family doctors in Brazil and other countries, aiming to discuss recent measures of the Ministry of Health that regulated the workload of physicians in the Family Health Care Strategy. The approach is taken from literature and Brazilian legislation on the subject, in a comparative perspective with experiences from other countries. The research revealed the existence of a pattern of poor state regulation of both medical labor and training in Brazil, especially with regard to family medicine, as compared with international experiences. This resulted in low supply of professionals for the Family Health Care Strategy and contributed to the recent flexibilization of workload of physicians and the creation of the Mais Médicos Program by the Ministry of Health. The option for flexibilization in place of more regulation on the profession can affect the integration of family health teams, which is a central element of the strategy and seemingly contradicts the emphasis on the priority of their role as repeatedly stated by the ministry.

> Key words: Brazilian Family Health Strategy; Family Health Medicine; health manpower; health care public policies. 